\title{
Clinical Research of Early Hyperbaric Oxygen Therapy on Patients with Hypertensive Cerebral Hemorrhage After Craniotomy
}

\author{
Xuejian WANG, Yang CHEN, Zhifeng WANG, Ming QIAN \\ Nantong University, The Second Hospital Affiliated to Nantong University, Department of Neurosurgery, Nantong, Jiangsu, PR China \\ Corresponding author: Xuejian WANG 6841441@163.com
}

\section{ABSTRACT}

AIM: To observe the effect of early hyperbaric oxygen (HBO) therapy on the improvement of consciousness and prognosis of patients with severe brain damages after craniocerebral craniotomy.

MATERIAL and METHODS: Eighty-one patients who had cerebral hemorrhage and underwent clearance of hematoma and decompressive craniectomy from August 2013 to August 2016 were retrospectively analyzed. The patients were divided into HBO and non-HBO therapy groups. The treatment effects were scored and subjected to corresponding statistical analysis.

RESULTS: There were significant differences in the Glasgow coma scale (GCS) scores at 3 and 5 weeks ( $t=2.293$ and $t=3.014$, respectively, $p<0.05)$, and in Glasgow outcome scale (GOS) scores at 5 weeks and 3 months between the two groups ( $<0.05$ ).

CONCLUSION: Early HBO therapy could improve the consciousness and prognosis of patients with cerebral hemorrhage after craniotomy.

KEYWORDS: Cerebral hemorrhage, Post-craniotomy, Hyperbaric oxygen therapy, Glasgow coma scale, Glasgow outcome scale

\section{INTRODUCTION}

$\mathrm{H}$ ypertensive intracerebral hemorrhage is an important cause of death and disability in patients worldwide (8). The clinical prognosis of patients with hypertensive intracerebral hemorrhage largely depends on the molecular biological and cytological changes of the secondary brain damages, and is related to the pathophysiology of brain damage $(1,2,8,15)$. After surgical treatment, patients with a large amount of bleeding still need take corticosteroids, calcium antagonists, endogenous brain protective drugs, and analeptic drugs as well as auxiliary treatments such as dehydration, mild hypothermia treatment, nutritional support therapy, rehabilitation training, and other comprehensive therapies. Among them, hyperbaric oxygen (HBO) is one of the most effective methods for postoperative rehabilitation, reducing mortality and improving neurological outcome $(3,4,21)$. This study was based on patients who had hypertensive intracerebral hemorrhage and were subjected to craniotomy hematoma plus decompressive craniectomy with/ without postoperative HBO therapy to compare the effects of $\mathrm{HBO}$ on the changes in Glasgow coma scale (GCS) scores and Glasgow outcome scale (GOS) scores and improvement of the consciousness and prognosis of these patients. We observed the effect of early HBO therapy on the improvement of consciousness and prognosis of patients with severe brain damages after craniocerebral craniotomy, and the results are reported as follows.

\section{MATERIAL and METHODS}

This research was approved by the Ethics Committee of the Second Hospital affiliated to Nantong University. Informed consent was obtained from all patients and this investigation was conducted according to the principles expressed in the Declaration of Helsinki. 


\section{Subjects}

The subjects were 104 inpatients who admitted to the emergency room of the Neurosurgery Department of the First People's Hospital of Nantong from August 2013 to August 2016 due to cerebral hemorrhage and were subjected to clearance of hematoma by craniotomy and decompressive craniectomy. Around 8 days (range: $7-12$ ) after craniotomy, these patients were assigned into either $\mathrm{HBO}$ or non-HBO therapy groups according to the random number table method. Of them, 52 patients were in the HBO therapy group and 52 patients were in the non-HBO therapy group. Among them, 12 patients in the HBO therapy group and 11 patients in the non-HBO therapy group did not finish the whole treatment course. Therefore, a total of 40 patients in the HBO therapy group and 41 patients in the non-HBO therapy group were enrolled in the study.

\section{Inclusion Criteria}

Patients were enrolled in the clinical study if they 1) were at ages of $18-60,2)$ had intracerebral hemorrhage and GCS score of 3-8 at admission, 3) were treated with craniotomy plus decompression surgery within 24 hours after admission, 4) had stable vital signs about 1 week after admission and could tolerate HBO therapy, and 5) did not have acute infection and chronic diseases such as hypertension, coronary heart disease, diabetes, chronic bronchitis, chronic anemia, etc. at admission. In addition, all patients and their family members signed the "Informed consent to treatment".

Patients were excluded if they 1) had previous neurological history such as cerebral infarction, cerebral hemorrhage, brain tumors, cerebral vascular malformations, cerebral aneurysms, Parkinson's disease, primary epilepsy, nervous system infections and other related nervous system diseases, 2) suffered from shock and had less than $90 \%$ oxygen saturation at admission, 3) were awake or dead, or had GOS=1 or GOS=5 before HBO therapy, 4) had infection, and chronic diseases such as hypertension, coronary heart disease, diabetes, chronic bronchitis, chronic anemia, etc. at admission, and 5) failed to complete HBO therapy and had severe special events during the course of treatment.

\section{Assignment}

According to randomized clinical trial protocol (random number table method), a total of 81 patients were enrolled in the study. Among them, 40 were assigned in the HBO therapy group and 41 were in non-HBO control group.

Among the 40 patients in HBO group, 29 were male and 11 were female. Their median age was 36 years old (18-58 years old). Their average body weight was $58.62 \pm 8.23 \mathrm{~kg}$. Their mean GCS score at admission was $6.2 \pm 1.63$ and their mean GCS score before HBO therapy was $7.1 \pm 1.58$. In addition, 26 underwent tracheotomy, and 27 had brain herniation at admission.

Among the 41 patients in non-HBO group, 30 were male and 11 were female. Their median age was 37 years old (1960 years old). Their average body weight was $60.12 \pm 7.23$ $\mathrm{kg}$. Their mean GCS score at admission was $6.3 \pm 1.56$ and their mean GCS score before HBO therapy was $7.3 \pm 1.35$.
In addition, 26 patients underwent tracheotomy, and 27 had brain herniation at admission.

\section{Treatments}

All the patients were treated with craniotomy plus decompressive craniectomy and subjected to postoperative electrocardiogram monitoring, blood oxygen monitoring and oxygen absorption monitoring at medium flow. Patients who need tracheotomy were subjected to tracheotomy within 4 days after operation and other supportive cares such as sputum suction, dehydration, hemostasis, stomach protection, infection prevention, nerve nutrition, energy and fluid supplement and other drugs. In addition, patients were subjected to CT at the day or the next day of operation. At stable condition about 3 days after surgery, patients were not given hemostatic drugs. At 5 days after surgery, patients were given blood-circulation activating drugs. And at about 7 days after admission, patients with stable disease conditions in the HBO group were subjected to two courses of HBO therapy, once a day for 10 days per course. HBO therapy was conducted using a medical air pressurized cabin. The treatment pressure was set at $0.2 \mathrm{MPa}$. Patients were subjected to the pressure for $20 \mathrm{~min}$, oxygen adsorption for $60 \mathrm{~min}$, rest for $10 \mathrm{~min}$ and final decompression for $20 \mathrm{~min}$. The treatment efficacy was observed and evaluated after 2 courses of the treatment.

\section{Observation Indicators}

The observation indicators included 1) GCS sores at admission and at 1, 3, and 5 weeks after admission; 2) GOS grading (GOS) before $\mathrm{HBO}$, at 5 weeks or the end of HBO therapy (GOS5w) and at 3 months after admission (GOS3m). GOS grading was divided into 5 grades. Patients who died were considered as Grade I, who survived in vegetative state were Grade 2, who were severely disabled were Grade 3, who were disabled were Grade 4, and who were in good conditions were Grade 5.

\section{Statistical Methods}

All patient information were collected, input and analyzed using the SPSS13.0 statistical analysis software. The measurement data with normal distribution were described as mean and standard deviation, and analyzed using two independent samples t-test. The count data were described as rate and analyzed using chi-square (f) test. $p<0.05$ was considered as statistically significant difference.

\section{RESULTS}

There were no statistically significant differences in gender, GCS scores at admission, GCS scores before and after HBO therapy between patients in the two groups (all p> 0.05). However, the GCS scores at 3 weeks and 5 weeks after admission were significantly different between the two groups of patients $(t=2.293$ and $t=3.014$, respectively, $p<0.05$ ). In addition, chi-square (f) test showed that the number of patients with increased GOS scores at 5 weeks and 3 months after admission were significantly different between the two groups of patients $(p<0.05)$. 
Table I: Average GCS Score of GCS3 and GCS5 in Both Groups

\begin{tabular}{|c|c|c|c|c|}
\hline \multirow{2}{*}{ Group } & \multirow{2}{*}{$\mathbf{n}$} & GCS & GCS 3 weeks & GCS 5 weeks \\
\hline & & prior treatment & after admission & after admission \\
\hline Hyperbaric oxygen (HBO) & 40 & $7.1 \pm 1.58$ & $11.2 \pm 2.89$ & $12.6 \pm 2.70$ \\
\hline Non-HBO therapy groups & 41 & $7.3 \pm 1.35$ & $9.81 \pm 2.56$ & $10.61 \pm 3.00$ \\
\hline $\mathrm{t}$ & & 0.613 & 2.293 & 3.014 \\
\hline $\mathrm{p}$ & & $>0.05$ & $<0.05$ & $<0.01$ \\
\hline
\end{tabular}

GCS3: GCS 3 weeks after admission, GCS5: GCS 5 weeks after admission.

Table II: Comparison with Gender and Improve of Two Phase GOS in the Two Groups of Patients

\begin{tabular}{|c|c|c|c|c|c|c|}
\hline \multirow{2}{*}{ Group } & \multicolumn{2}{|c|}{ Sex } & \multicolumn{2}{|c|}{ iGOS5w } & \multicolumn{2}{|c|}{ iGOS3m } \\
\hline & $\mathbf{M}$ & $\mathbf{F}$ & $\mathbf{N}$ & $\mathbf{Y}$ & $\mathbf{N}$ & $\mathbf{Y}$ \\
\hline Hyperbaric oxygen (HBO) & 30 & 11 & 26 & 15 & 21 & 20 \\
\hline Non-HBO therapy groups & 29 & 11 & 17 & 23 & 12 & 28 \\
\hline$\chi^{2}$ & \multicolumn{2}{|c|}{0.001} & \multicolumn{2}{|c|}{3.556} & \multicolumn{2}{|c|}{3.776} \\
\hline $\mathrm{p}$ & \multicolumn{2}{|c|}{$>0.05$} & \multicolumn{2}{|c|}{$<0.05$} & \multicolumn{2}{|c|}{$<0.05$} \\
\hline
\end{tabular}

GOS: Glasgow outcome scale, M: male, F: female, N: no, Y: yes, iGOS5w: The increase numbers of patients' GOS 5 weeks later compared to patients when starting treatment, iGOS3m: The increase numbers of patients' GOS 3 month later compared to patients when starting treatment.

\section{DISCUSSION}

Hypertensive intracerebral hemorrhage is an intracranial hemorrhage caused by cerebral vascular rupture. Because of its high mortality and morbidity, it has been plaguing clinicians (8). Timely surgery is one of the effective treatments and can effectively save the lives of patients and minimize secondary nerve injury due to hemoglobin dissolution $(13,15)$. However, even with active treatment, neurological deficits are still high and mainly related to cerebral edema due to cerebral hemorrhage and the surrounding tissue damage $(19,20)$. HBO is currently considered to have a beneficial effect on treatment of cerebral hemorrhage. HBO has been used for more than two hundred years. Due to its unique therapeutic effect, HBO therapy is called a treatment of disease searching (6). A large number of worldwide studies showed that the action mechanism of $\mathrm{HBO}$ is more complex and corresponding to the pathophysiological changes of cerebral hemorrhage $(4,8,15,21)$.

The brain tissue damages after intracerebral hemorrhage include two aspects: direct bleeding-induced injury, and secondary brain tissue injury. HBO showed protection of cerebral injury at different stages after cerebral hemorrhage. Worldwide studies have shown that 1) HBO can make cerebral vasoconstriction, thereby reducing CBF, ICP, and cerebral edema, while expand blood vessels at the ischemic area, accelerating the blood flow velocity, opening more micro-vessels and promoting the establishment of collateral circulation $(10,12)$; 2) HBO can enhance the expression of brain-derived nerve growth factor in brain cells, thereby promoting nerve axon growth; 3) HBO can significantly reduce the levels of serum cell adhesion molecules and matrix metalloproteinase-9, and blood viscosity, while increase red blood cell deformability $(12,14)$; 4) HBO can increase brain oxygen supply, protect mitochondria, improve the utilization of glucose and the level of aerobic metabolism in the patients with severe brain injury at the level of mitochondria, promote oxygen metabolism and improve oxygen utilization (5); 5) HBO can increase the activity of antioxidant enzymes, and scavenge oxygen free radicals; 6) HBO can promote cognitive recovery, increase brain ATP level, and reduce hippocampal nerve cell loss induced by hypoxia; 7) HBO can reduce leukocyte infiltration and the level of inflammatory cytokines, inhibit inflammatory cell activity, and maintain blood-brain barrier permeability; 8) HBO can enhance phagocytic and plasmin activities, and accelerate hematoma removal rate; 9) HBO can improve aerobic metabolism and energy supply and maintain normal physiological functions of brain cells and vascular endothelial cells (12); 10) HBO can increase plasma oxygen dissolution, and promote long-distance oxygen supply even if the vessel is completely blocked $(9,12)$; and 11) HBO can promote the restoration of blood-brain barrier integrity after vascular infarction in rabbits (17).

HBO therapy may have the following side effects. First, Yasu reported that $\mathrm{HBO}$ might promote free oxygen radical production and lipid peroxidation in normal rats (22). However, Mink and Dutka showed that although HBO increased free radicals, it was not associated with lipid peroxidation and did not affect neurological recovery (16). Second, some scholars believed that HBO could cause vasospasm and contraction, therefore reducing blood supply. However, multiple studies have confirmed that although $\mathrm{HBO}$ induced vasoconstriction and blood flow reduction, it did not lead to clinical damage, 
and SPECT imaging prompted that brain tissue metabolism was not affected $(16,18)$. It has been hypothesized that increased oxygen supply from HBO may be sufficient to counteract its side effects such as reduced cerebral blood flow (12), the phenomenon similar to the steal phenomenon reported by Lassen and Palvogyi (11), and blood supply increase in the injured area resulted from reactivity due to normal vasoconstriction.

In the present study, the GCS scores of patients in the two groups showed the same trend at the third week and the fifth week after treatment, but the improvement of the HBO group was better than that of the non-HBO group, indicating that early HBO therapy had better effects on improvement of consciousness. Similarly, the GOS scores of patients in both groups improved after 5 weeks and 3 months of treatment, indicating that HBO therapy could improve the prognosis and the living status of patients after cerebral hemorrhage. All these results suggest that HBO has a very good role in promoting rehabilitation of patients with cerebral hemorrhage after surgery. But it should be noted that HBO is one of the measures to promote rehabilitation and needs to be combined with other treatments in order to achieve the best therapeutic effect, minimize brain damage and maximize patient recovery. Experimental animal studies suggest that $\mathrm{HBO}$ combined with nicardipine can promote neurological rehabilitation (7). Further study and summary of HBO therapy program are needed to better promote patient recovery.

\section{CONCLUSIONS}

The following conclusions could be drawn from our results. First, early HBO therapy could promote awareness of patients with cerebral hemorrhage after craniotomy. Second, early HBO therapy could improve the prognosis of patients with cerebral hemorrhage after craniotomy. Third, early HBO therapy in combination with other rational rehabilitation measures can effectively promote the rehabilitation of patients, minimize the extent of their disability, and improve their social life. Overall, early HBO therapy has immeasurable beneficial effects.

\section{- ACKNOWLEDGEMENTS}

This research supported by Young Medical Project of Nantong City Health Bureau (WQ2014016), Traditional Chinese Medicine Science and Technology Project in Jiangsu Province (No. YB2015113), and (3) the Science and Technology Program of Nantong City (No. MS12015016).

\section{REFERENCES}

1. Cao H, Ju K, Zhong L, Meng T: Efficacy of hyperbaric oxygen treatment for depression in the convalescent stage following cerebral hemorrhage. Experimental and Therapeutic Medicine 5(6):1609-1612, 2013

2. Chen CH, Manaenko A, Zhan Y, Liu WW, Ostrowki RP, Tang J, et al: Hydrogen gas reduced acute hyperglycemia-enhanced hemorrhagic transformation in a focalischemia rat model. Neuroscience 169(1):402-414, 2010
3. Chiu CD, Chen CC, Shen CC, Chin LT, Ma HI, Chuang HY, et al: Hyperglycemia exacerbates intracerebral hemorrhage via the downregulation of aquaporin-4: Temporal assessment with magnetic resonance imaging. Stroke 44(6):1682-1689, 2013

4. Chu H, Tang Y, Dong Q: Protection of granulocytecolony stimulating factor to hemorrhagic brain injuries and its involved mechanisms: Effects of vascular endothelial growth factor and aquaporin-4. Neuroscience 260:59-72, 2014

5. Contreras FL, Kadekaro M, Eisenberg HM: The effect of hyperbaric oxygen on glucose utilization in a freezetraumatized rat brain. J Neurosurg 68:137-141, 1988

6. Gabb G, Robin ED: Hyperbaric oxygen: A therapy in search of diseases. Chest 92:1074-1082, 1987

7. Iwatsuki N, Takahashi M, Ono K, Tajima T: Hyperbaric oxygen combined with nicardipine administration accelerates neurologic recovery after cerebral ischaemia in a canine model. Crit Care Med 22(5):858-863, 1994

8. Jing Wu, Jiong Chen, Hua Guo, Fang Peng: Effects of highpressure oxygen therapy on brain tissue water content and AQP4 expression in rabbits with cerebral hemorrhage. Cell Biochem Biophys 70:1579-1584, 2014

9. Kanno T, Nagata J, Nonomura K, Asai T, Inoue T, Nakagawa T, Mitsuyama F: New approaches in the treatment of hypertensive intracerebral haemorrhage. Stroke 24 Suppl 12: 196-100, discussion 1107-8, 1993

10. Kohshi K, Yokota A, Konda N, Kinoshita Y, Kajiwara H: Intracranial pressure responses during hyperbaric oxygen therapy. Neurol Med Chir (Tokyo) 31(9):575-581, 1991

11. Lassen NA, Palvogyi R: Cerebral steal during hypercapnia observed by the 133-Xenon technique in man. Scan J Clin Lab Inves Suppl 102:XIII-D, 1968

12. Lim J, Lim WK, Yeo TT, Sitoh YY, Low E: Management of haemorrhagic stroke with hyperbaric oxygen therapy - a case report. Singapore Med J 42(5):220-223, 2001

13. Matchett GA, Martin RD, Zhang JH: Hyperbaric oxygen therapy and cerebral ischemia: Neuroprotective mechanisms. Neurological Research 31(2):114-121, 2009

14. Mathieu D, Coget J, Vinakeir L, et al: Filtrabilite erythrocytaire et oxygenotherapie hyperbare. Circulation et metabolisme du Verveau 2:27-31, 1985

15. Michel-Monigadon D, Bonny C, Hirt L: C-Jun N-terminal kinase pathway inhibition in intracerebral hemorrhage. Cerebrovascular Diseases 29(6):564-570, 2010

16. Mink RB, Dutka AJ: Hyperbaric oxygen after global cerebral ischaemia in rabbits does not promote lipid peroxidation. Crit Care Med 23(8):1398-1404, 1995

17. Mink RB, Dutka AJ: Hyperbaric oxygen after global cerebral ischaemia in rabbits reduces brain vascular permeability and blood flow. Stroke 26(12):2307-2312, 1995

18. Neubauer RA, James P: Cerebral oxygenation and the recoverable brain. Neurol Res 20 Suppl I: S33-36, 1998

19. Ostrowski RP, Zhang JH: Hyperbaric oxygen for cerebral vasospasm and brain injury following subarachnoid hemorrhage. Translational Stroke Research 2(3):316-327, 2011 
20. Qing WG, Dong YQ, Ping TQ, Lai LG, Fang LD, Min HW, et al: Brain edema after intracerebral hemorrhage in rats: The role of iron overload and aquaporin 4. Journal of Neurosurgery 110(3):462-468, 2009

21. Ritzenthaler T, Dailler F, Vukusic S, Confavreux C, Marignier R: First attack of Devic's neuromyelitis optica following endovascular treatment and rupture of brain arteriovenous malformation. Multiple Sclerosis 17(7):895-896, 2011

22. Yasu $\mathrm{T}: \mathrm{H} 2 \mathrm{O} 2$ generation in rat brain in vivo correlates with oxygen pressure. Masui (English abstract) 35:1077-1082, 1986 